\title{
TEXTURE EVOLUTION AND YIELD LOCI IN FERRITIC STEELS
}

\section{N. MINGOLO and A.A. POCHETTINO}

Dpto. Ciencia de Materiales, Gcia. Desarrollo, CNEA Av. Libertador 8250, 1429 Buenos Aires, Argentina.

\section{INTRODUCTION}

Changes induced by different deformation paths in the texture and consequently, in the Yield Loci of ferritic steels are studied in this work. Crystalline rotations were predicted by using the classical Taylor Model assuming pencll glide deformations in grains (TPG) ${ }^{1}$ and the viscoplastic mode 1 with $\{110\}\langle 111\rangle,\{112\}\langle 111\rangle$ and $\{123\}\langle 111\rangle$ crysta lographic sitp systems under the full constraints (VFC) and the relaxed constrains (VRC) Taylor assumptions ${ }^{2}$.

Texture evolution and the corresponding Yield Loci were predicted for different deformation paths: rolling and rolling combined with equibiaxial expansion, plane tensile deformations, pure shear and uniaxial tensile tests. In the latter case, calculations take into account the plastic anisotropy of the steels, described by the contraction coefficient $q=-\left(E_{22} / E_{11}\right)$.

\section{TEXTURE PREDICTIONS}

Texture evolution predictions for different deformation paths were performed considering:

- An initial isotropic set of 552 orientations for rolling simulation. In the case of combined paths, the rolling texture used in calculations was that corresponding to a deformation $E=1$.

- Viscoplastic texture simulations were performed using a strain rate sensitivity value $m=0.05$, which gives a lowrate sensitivity solution to the single crystal constitutive equation and minimizes the computation effort ${ }^{3}$. 
- Isotropic hardening in grains was assumed for texture and Yield Loci evolutions predictions.

\section{RESULTS}

Texture simulations show that VRC and TPG models give the best aggreement with experimental pole figures, which are obtained from our experiments (Al killed steel and AISI 409 stainles stee1) and from 1iterature. The most important results obtained in our calculations can be summarized as follows:

Rolling: Fig. 1 shows the calculated $\{110\}$ Pole Figures (PF) for a thick reduction of $60 \%(E=1)$ using the TPG, VFC and VRC formulations. They are in agreement with the experimental $\{110\}$ PF, Fig. 1.d, corresponding to an Al Killed Steel (AKS). Calculated texture can be associated to the $\{111\}\langle U v W\rangle$ and the $\{h k 1\}\langle 110\rangle$ fibers, presenting reinforcements around the orientations $\{111\}\langle 110\rangle,\{111\}\langle 112\rangle$, $\{100\}\langle 110\rangle$ and $\{112\}\langle 110\rangle$. VRC presents the best agreeement with experimental PF. TPG formulation predicts an important reinforcement of the $\{111\}\langle 110\rangle$ component, while the VFC description tends to reinforce the $\{112\}\langle 110\rangle$ component (not observed in experimental PF). The latter description also has difficulties for the description of texture secondary components. Our VRC predictions are in agreement with those of Gilormini4, even if we have obtained a less sharper distribution of orientations.

Rolling + Untaxial Tenstle Tests: Calculations were performed considering tenstle tests along the rolling and transverse directions and the corresponding contraction coefficient values (q) associated to the stress states. Our experimental determinations of these coefficients show that, in the studied steels, their values can be considered as constants during the tensile test. Calculated and experimental textures present a good agreement, as it can be observed in Fig. 2.a-b for an AKS sample after a deformation of $E=0.12$ along the rolling direction of the sheet. PF were predicted using the TPG model. Related to rolling texture, a reinforcement of the $\{111\}\langle 110\rangle$ texture component is observed. In the case of a tensile test along TD ( $E=0.33$ ), the $\{111\}\langle 112\rangle$ component is reinforced in the predicted PF (VRC formulation), Fig. 2.c.. This result is coincident with that corresponding to Arminjons.

Rolling + Biaxial Expansion: Comparing with rolling textures, predictions of texture evolution after a biaxial expansion test ( $E=0.25)$ show a reinforcement of the $\{111\}$ (uvw) fiber component, spectally around the $\{111\}\langle 112\rangle$ ortentation, and a diminution of the $\{h k 1\}\langle 110\rangle$ fiber, except for 
orientations placed near the $\{111\}\langle 110\rangle$ and $\{100\}\langle 110\rangle$ components. TPG model calculations give the sharpest values of the calculated pole densities ${ }^{6}$.

Rolling + Uniaxial Compression: Calculations were performed considering a compression test along the roling direction with a contraction coefficient $q=0.6$ and a total deformation $E=0.33$. Predictions indicate a reinforcement of the fiber $\{111\}\langle u v w\rangle$, specially around the components $\{111\}\langle 112\rangle$. Particularly, the VRC model also predicts the existence of $\{h k 1\}\langle 110\rangle$ components placed near the $\{112\}\langle 110\rangle$ orientation, Fig. 3.a.

Rolling + Pure Shear: Texture calculation for pure shear ( $q=1, E=0.25$, rolling direction) shows a development of the \{111\}<uvw> fiber, spectally in the case of TPQ predictions, where a reinforcement of the $\{111\}\langle 110\rangle$ and $\{111\}\langle 112\rangle$ components can be observed, Fig. 3.b.

\section{YIELD LOCI EVOLUTIONS}

Fig. 4 shows the yield loct calculated using: a) the rolling texture simulated by using the VRC model (522 orientations) and b) the texture description of an AKS sheet given by the Orientation Distribution Function, which is obtained from experimental pole figures. These loct are in good agreement with experimental points and tangents obtained from tensile, compression and biaxial expansion tests ${ }^{6}$.

Taking into account the isotropic hardening hypothesis, texture evolution can only aiter the loci shape. Then, yield loci can be directly superposed in a $k$ representation in order to determine texture effects. In general, small changes in Yield Loci shape were determined for different deformation paths as biaxial expansion ( $E=0.25$ and $E=0.5)$ and unfaxial tensile and compression tests along the rolling and the transverse directions. As an example, Fig. 5.a shows the shape changes introduced by an uniaxtal compression test along the roliting direction $(E=0.33)$, whtch is related to the development of the fiber $\{$ hk 1$\}\langle 110\rangle$ predicted by the VRC.

Another interesting case is that corresponding to the loci changes induced by pure shear deformation. In this case, Fig. 5.b, an increment of yield stresses in the first quadrant, specially for the region associated to $q=0 \quad\left(E_{22}=0\right)$ can be observed. This change in the locus shape, associated to the reinforcement of the $\{111\}\langle u v w\rangle$ texture components, benefits the performance during deep drawing operations ${ }^{6}$. 

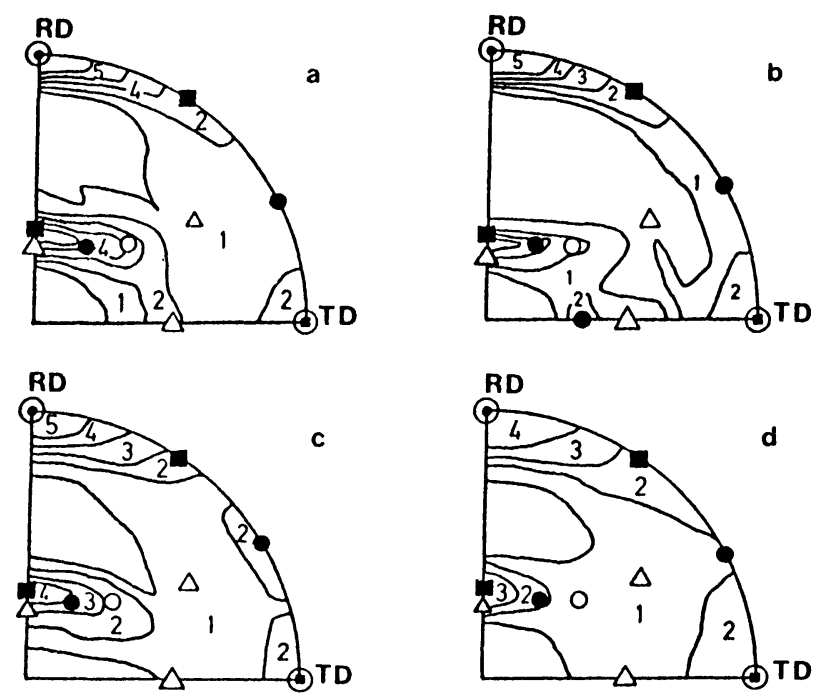

Figure 1: $\{110\}$ Pole Figures for rolling ( $E=1)$, calculated using (a) VRC, (b) VFC, (c) TPG and (d) experimental (AKS).
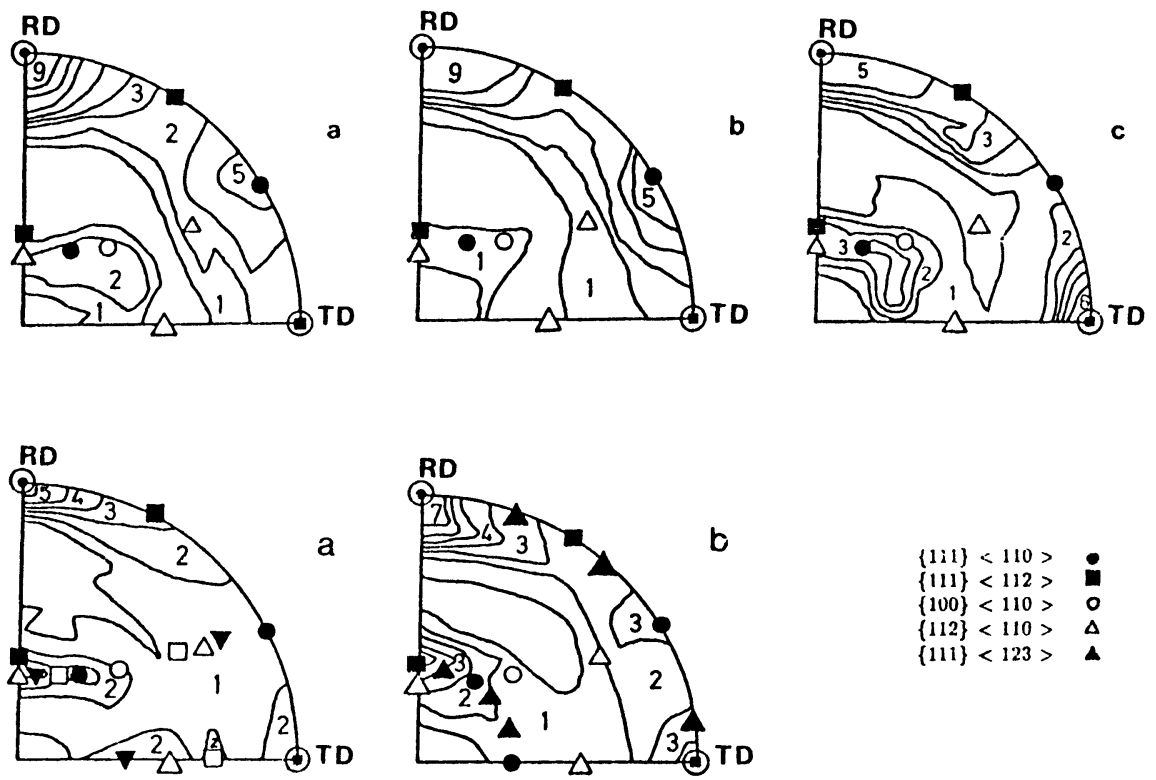

Figure 2: $\{110\}$ P.F. for rolling $(E=1)+$ uniaxial tenstle test (a) along RD ( $E=0.12$ ), TPG predictions and (b) experimental (AKS); (c) along TD ( $E=0.33$ ) predicted by VRC.

Figure 3: $\{110\}$ P.F. calculated for rolling $(E=1)+$ uniaxial compression along RD ( $E=0.33$ - VRC) and (b) rolling + pure shear ( $E=0.25-T P G)$. 


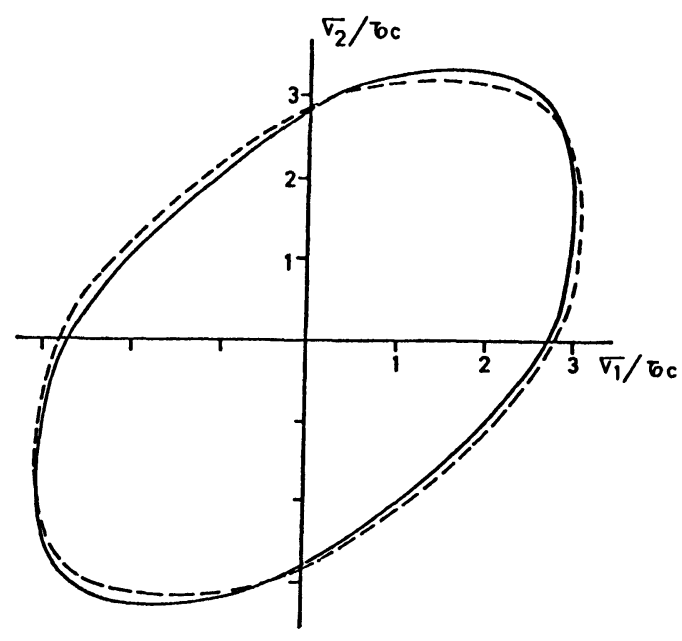

Figure 4: Yield loci (a) - Calculated from rolling texture simulated by using VCR model ( $E=1)$, (b) -- Calculated from the experimental rolling texture of an Al killed Steel.
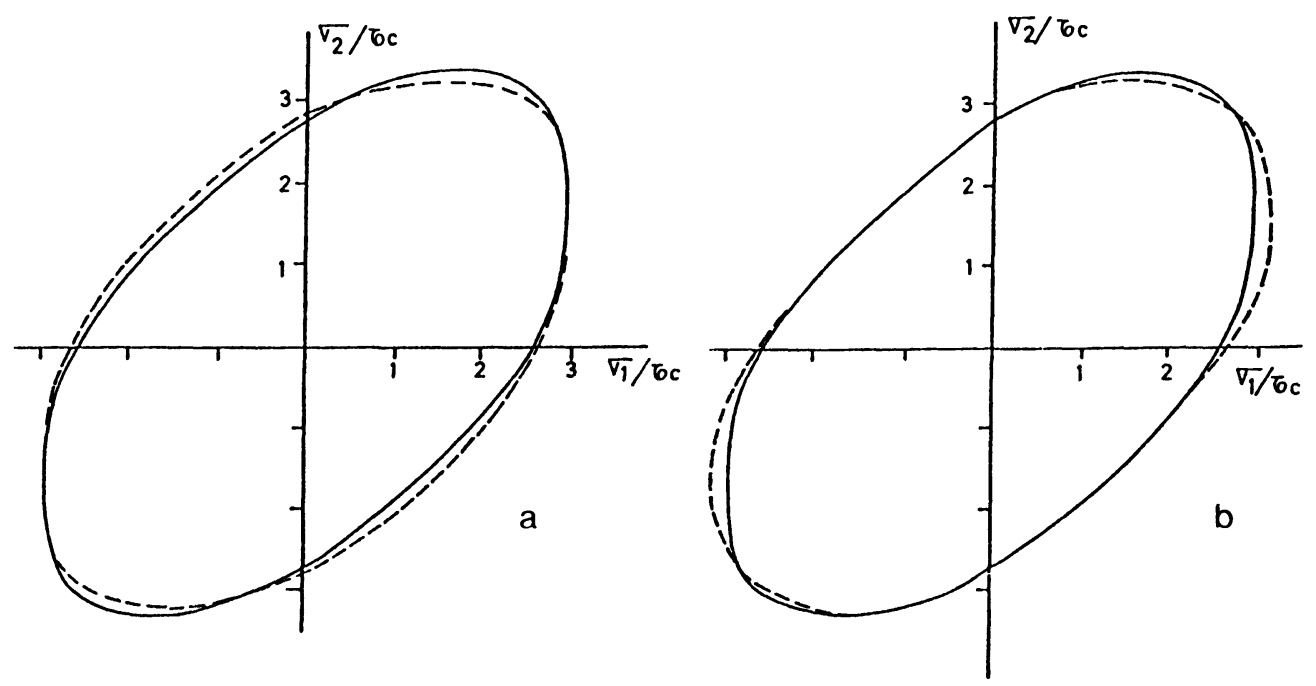

Figure 5: Yield loci evolutions related to rolling - (VCR model $E=1$ ) (a) Compression a long RD ( $E=0.33$, VCR mode 1 ), (b) pure shear ( $E=0.25$, TPG mode 1 ). 


\section{DISCUSSION}

As it was mentioned above, texture evolution described by VRC and TPG presents the best agreement with experiments. Particularly, for heavy rolling reductions (E>1.), it was shown that the VRC model presents the best agreement. A detailed study of the possible active slip systems and the activity on each one shows that in high strained grains the possible slip planes determined by the TPG tend to coincide with the crystallographic planes $\{123\},\{112\}$ and $\{110\}$, which are used in the VCR descriptions. As the activation of slip systems is low dependent of the strain rate sensitivity, the boundary conditions impossed in grains by the VCC model are responsible of the best texture predictions.

The fact that texture evolution under different deformation paths does not introduce significant changes in the shape of the predicted Yield Loci can be related: 1) to the facility for the accommodation of plastic deformation in grains by means of the $\{123\}\langle 111\rangle,\{112\}\langle 111\rangle$ and $\{110\}\langle 111\rangle$ slip systems (VRC mode1) or \{hk 1$\}\langle 111\rangle$ pencil gitde (TPG description); 2) to the isotropic hardening of the analysed materials. Results of uniaxial tensile and compression tests in different directions of the sheet as well as those corresponding to equibiaxial expansion tests performed in the Al killed and the AISI 409 stainless steel show that the strain hardening coefficients present similar values.

ACKNOWLEDGEMENTS: This work was partially supported by the PMM OEA-CNEA.

\section{BIBLIOGRAPHY}

1. F. Royer, C. Tavard and P. Penning, J. App. Cryst., 12, 436 (1979).

2. A. Molinari, G.R. Canova and S. Ahzi, Acta Metall., 25, 2983 (1987).

3. A.D. Rollet and U.F. Kocks, Proc. Eighth Int. Conf. on Textures of Materials, (J.S. Kailend and G. Gottstein Eds., The Metallurgical Soc, 1988), p. 375.

4. P. Gillormini, Acta Metali., in press.

5. M. Arminjon, IRSID Report-RE 1186 (1985).

6. N. Mingolo, Ph.D. Thesis (Untv. Nac. Buenos Atres, Argentina) 1990.

7. N. Mingolo, A.A. Pochettino, M. Thomas and R. Penelle, Proc. Eighth Int. Conf. on Texture of Materials, (J.S. Kallend and G. Gottstein Eds., The Metal1. Soc., 1988) p. 1059. 8. N. Mingolo and A.A. Pochettino, to be published. 